\title{
Acute Lower Limb Ischemia Secondary to COVID-19: A Case Report
}

\author{
Komal Gharsangi ${ }^{1}$, Rajesh Bhawani ${ }^{2}$, Nitesh Kumar ${ }^{3}$, Rohini Thakur ${ }^{4}$ \\ ${ }^{1}$ Assistant Professor, ${ }^{2}$ Prof and Head, ${ }^{3}$ Senior Resident, Department of General Medicine, Shri Lal Bahadur \\ Shastri Govt Medical College, Mandi, Himachal Pradesh-175008 \\ ${ }^{4}$ Senior Resident, Department of Radiodiagnosis, Shri Lal Bahadur Shastri Govt Medical College, Mandi, \\ Himachal Pradesh-175008 \\ Corresponding Author: Rajesh Bhawani
}

\begin{abstract}
COVID -19 is not just a respiratory illness; it is a great masquerader with clinical manifestations from pneumonia, acute respiratory distress syndrome to endothelial dysfunction, hypercoagulability and multiorgan failure. Thrombotic complications due to Covid related coagulopathy is of concern as it further leads to poor clinical outcome in severe cases of Covid 19. Acute limb ischemia (ALI) is a rare manifestation of Covid related coagulopathy.
\end{abstract}

Key words: COVID, coagulopathy, acute limb ischemia (ALI)

\section{INTRODUCTION}

Since COVID-19 pandemic was first identified in Wuhan, China in December 2019, it has spread globally to 200 countries. By May 2021 the total number of COVID 19 cases in India crossed 20 million with number of deaths more than 3 lakhs. SARS-COV2, the etiological agent, once goes deep inside the body affects not only lungs but many other organs including heart, liver, kidneys, brain , blood vessels. The damage to the lungs is accompanied by hyperimmune response to release cytokine storm which is said to be the cause of sudden deterioration in the patients. Vascular thrombotic manifestation due to abnormal clotting also plays a role in lethal COVID -19. The clotting in vasculature is associated with increased risk for complications like deep vein thrombosis, pulmonary embolism, ischemic strokes, acute coronary syndrome.

There have been many explanations for this abnormal clotting like inflammatory cytokine IL-6 in particular is responsible for the vascular complication by expressing tissue factor on monocytes and macrophages leading to thrombin generation. Apart from this endothelium plays an essential role in maintaining a dynamic interplay between the procoagulant and fibrinolytic factors in the vascular system. In the quiescent state, the endothelium forms a barrier between the prothrombotic subendothelial layer and procoagulant factors in the blood. In times of stress, activated endothelial cell express more plasminogen activator inhibitor-1, tissue factor and release von Willebrand factor. Activated endothelial cells lower activity of thrombomodulin and tissue plasminogen activator, favouring thrombus accumulation. $^{1}$ Another cause for pathogenesis of coagulation includes hypoxia. In conditions with severe COVID19, hypoxia can trigger thrombosis by increasing hypoxia-inducible transcription factor-dependent signaling pathway as well as by increasing blood viscosity. ${ }^{2}$ 


\section{CASE HISTORY}

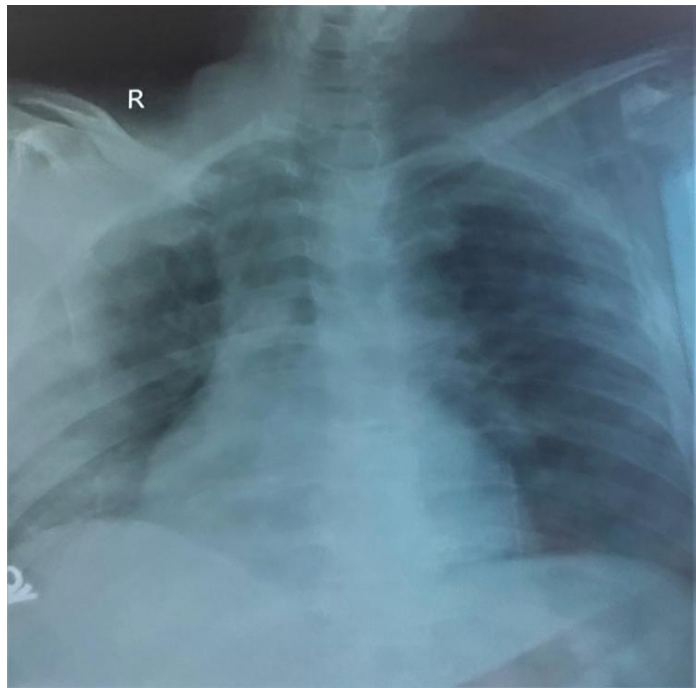

Figure1: Chest Xray showing bilateral opacity

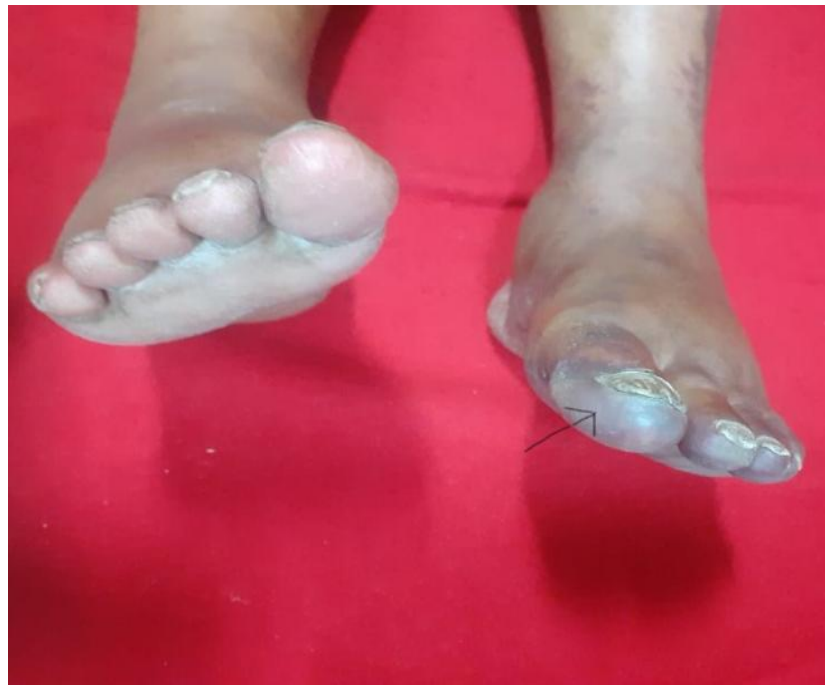

Fig2. Left foot showing bluish discoloration of the toes (arrow marked).
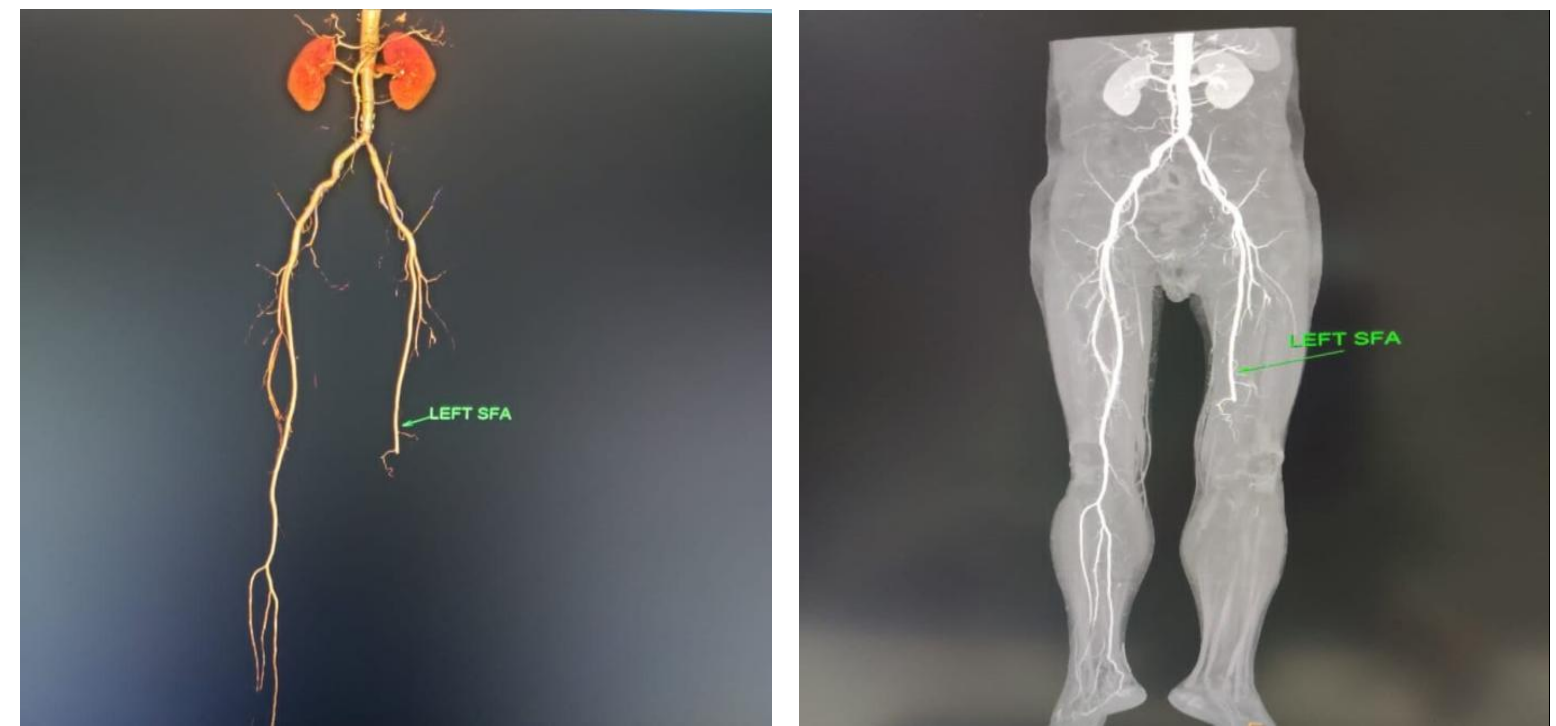

Fig 3 ARTERIAL OCCLUSION OF LOWER LIMB IN COVID 19: CT angiography showing occlusion of left superficial femoral artery $12 \mathrm{~cm}$ above knee joint. E: Angiogram showing no flow in the distal left leg.

A 52-year-old male without any other co-morbid illness presented to the hospital with primary complaints of shortness of breath. His diagnosis of COVID-19 infection was made 1 day prior to the presentation. His initial symptoms were of fever, cough which started 5 days prior to the day of his COVID-19 diagnosis. On admission, he was afebrile, respiratory rate of 28 breaths per minute, saturation of 80 percent on room air. Patient was put on venturi mask after which the saturation became stable at $94 \%$. Chest x-ray showed peripheral pulmonary opacities and consolidations bilaterally (Fig1). Patient was started on low molecular weight heparin, dexamethasone, remdesivir, antibiotics on day of admission. Electrocardiogram showed normal sinus rhythm without any signs of ischemia. On the second day of admission patients started complaining of severe left lower limb pain. On examination the left foot was cold and there was marbling of forefoot and toes (Fig2). The peripheral pulses were absent. He underwent CT angiogram which showed complete occlusion of left superficial femoral artery and $60 \%$ luminal compromise of left common iliac artery (Fig 3). His blood investigations revealed leucocytosis, $\mathrm{ESR}=50 \mathrm{~mm}, \mathrm{CRP}=35, \mathrm{D}-$ Dimer $=1.7 \mathrm{mg} / \mathrm{L}$. In view of urgent surgical 
intervention patient was shifted to CTVS department for embolectomy.

\section{DISCUSSION}

Acute lower limb ischemia is a vascular emergency with threat to limb viability. Arterial embolism is the most common cause of acute limb ischemia. COVID 19 and its association with coagulation abnormality have led to both venous and arterial thrombotic events. Klok et al. conducted a study on 184 patients with COVID-19, where the incidence of thrombosis was $31 \%$ overall, while $27 \%$ of cases were of venous thromboembolism and $3.7 \%$ showed arterial thrombotic events. ${ }^{3}$ In Italy, an observational study was performed to investigate the incidence and outcomes of 20 patients with acute limb ischemia which showed that the incidence of acute limb ischemia to be greater in the previous few months of 2020 compared with 2019, and they attributed the higher incidence to the increase in cases caused by thromboembolic disease associated with COVID-19. ${ }^{4}$

Although arterial thrombosis involving upper and lower extremity is being noted among COVID-19 patients, multiple retrospective analysis have demonstrated incidents of thrombosis ranging from $12 \%$ to $31 \%$ with a minority of these events are arterial. Any arterial segment can be involved in this condition, there have been reports of arterial thrombosis of brachial artery, radial artery, aorta, iliac and femoral arteries, superior mesenteric artery and even prosthetic vascular grafts as well. ${ }^{5}$ A single centre study performed at King Abdullah University Hospital in the north of Jordan observed that prevalence of acute lower limb ischemia in patients infected with COVID-19 was $0.54 \%{ }^{6}$

\section{CONCLUSION}

Coronavirus disease 19 with varied spectrum of manifestation stands a problem in management of critically ill patients. As thought initially that COVID was just a lung pathology has been overshadowed by additional theories of vasculopathy with clotting abnormalities. It is important that health care workers are aware of different life threatening thrombotic manifestations, like acute limb ischemia (ALI) in our case report, so that it is detected and treated on time.

\section{Acknowledgement: None}

\section{Conflict of Interest: None}

\section{Source of Funding: None}

\section{REFERENCES}

1. Wang M., Hao H., Leeper N.J., Zhu L. Thrombotic regulation from the endothelial cell perspectives. Arterioscler Thromb Vasc Biol Jun 2018;38:e90-e95

2. Tang N, Bai H, Chen X, Gong J, Li D, Sun Z. Anticoagulant treatment is associated with decreased mortality in severe coronavirus disease 2019 patients with coagulopathy. J Thromb Haemost.2020 Mar27;18:1094-1099

3. Klok F, Kruip M, van der Meer N, et al .Incidence of thrombotic complications in critically ill ICU patients with COVID19.Thromb Res. 2020;191:145-147.

4. Ahmad H, Shubair SM, Kruer J, Hatoum CA. Acute lower-extremity ischemia in a patient with COVID-19. Am J Case Rep. 2020;21:928471

5. Veerasuri S, Kulkarni SR, Wilson WR, Paravastu SCV. Bilateral acute lower limb ischemia secondary to COVID-19. Vasc Endovascular Surg. 2020;55(2):196-199.

6. Nabil Al-zoubi, Nawaf Shatnawi, and Hamza Jarbo.Acute Lower Limb Ischemia in Patients Infected with COVID-19.Int J Gen Med.2021 Mar 11;14:833-839

How to cite this article: Gharsangi K, Bhawani $\mathrm{R}$, Kumar $\mathrm{N}$ et.al. Acute lower limb ischemia secondary to COVID-19: a case report. Int J Health Sci Res. 2021; 11(6): 161-163. DOI: https://doi.org/10.52403/ijhsr.20210625 